\title{
Familial 1.3-Mb 11p15.5p15.4 Duplication in Three Generations Causing Silver-Russell and Beckwith-Wiedemann Syndromes
}

\author{
Mari-Anne Vals ${ }^{a, b}, d$ Tiina Kahre ${ }^{a, d}$ Pille Mee $^{c}$ Kai Muru $^{a}$ Eha Kallas ${ }^{b} d$ \\ Olga Žilina ${ }^{a, e}$ Vallo Tillmann ${ }^{b, d}$ Katrin Õunap ${ }^{a, d}$ \\ ${ }^{\mathrm{a}}$ Department of Genetics, ${ }^{\mathrm{b}}$ Children's Clinic, and ' United Laboratories, Tartu University Hospital, and \\ Departments of ${ }^{d}$ Pediatrics and ${ }^{~}$ Biotechnology, Institute of Molecular and Cell Biology, University of Tartu, \\ Tartu, Estonia
}

\section{Key Words}

11 p15 duplication 11 p15 imprinting disorders . Beckwith-Wiedemann syndrome $\cdot$ Imprinting control region $1 \cdot$ Imprinting control region 2 . Silver-Russell syndrome

\begin{abstract}
Silver-Russell syndrome (SRS) and Beckwith-Wiedemann syndrome (BWS) are 2 opposite growth-affecting disorders. The common molecular cause for both syndromes is an abnormal regulation of genes in chromosomal region $11 \mathrm{p} 15$, where 2 imprinting control regions (ICR) control fetal and postnatal growth. Also, many submicroscopic chromosomal disturbances like duplications in 11 p15 have been described among SRS and BWS patients. Duplications involving both ICRs cause SRS or BWS, depending on which parent the aberration is inherited from. We describe to our knowledge the smallest familial pure 1.3-Mb duplication in chromosomal region $11 \mathrm{p} 15.5 \mathrm{p} 15.4$ that involves both ICRs and is present in 3 generations causing an SRS or BWS phenotype.
\end{abstract}

(c) 2015 S. Karger AG, Basel

Silver-Russell syndrome (SRS, OMIM: 180860) is a clinical disorder that primarily includes pre- and postnatal growth disturbances with normal head circumference, along with other distinctive characteristics like asymmetry of body and limbs, craniofacial features, and 5th finger (F5) clinodactyly. Beckwith-Wiedemann syndrome (BWS, OMIM: 130650) is also a growth-affecting disorder which causes overgrowth with many additional clinical features like macroglossia, organomegaly, and increased risk of childhood tumors [Weksberg et al., 2010]. The most common molecular cause for both syndromes is an abnormal regulation of genes in chromosomal region 11p15 [Gicquel et al., 2005], where 2 imprinting control regions (ICR1 and ICR2) control fetal and postnatal growth. ICR1 contains the maternally expressed H19 gene and the paternally expressed IGF2 gene, whereas ICR 2 contains the maternally expressed KCNQ1 and $C D K N 1 C$ genes and the paternally expressed KCNQ1OT1 gene. Normally, genes that are expressed in 1 allele are imprinted (methylated) and silenced in the other allele. Imprinting disturbances lead to abnormal expression of these genes and the clinical phenotypes of SRS or BWS. Approximately $40 \%$ of SRS patients show hypomethylation of ICR1, whereas up to $50 \%$ of BWS patients have ICR2 hypomethylation [Eggermann et al., 2008]. In addition, in SRS and BWS patients, numerous submicroscopic chromosomal disturbances have been described, among them duplications, deletions, inversions and/or translocations affecting chromosome 11p15 [Begemann et al., 2012; Fokstuen and Kotzot, 2014]. Large duplica-

\section{KARGER 125}

(c) 2015 S. Karger AG, Base

$1661-8769 / 15 / 0063-0147 \$ 39.50 / 0$

E-Mail karger@karger.com

www.karger.com/msy
Mari-Anne Vals

Department of Genetics

Tartu University Hospital

2 L. Puusepa Street, Tartu 51014 (Estonia)

E-Mail mari-anne.vals@kliinikum.ee 


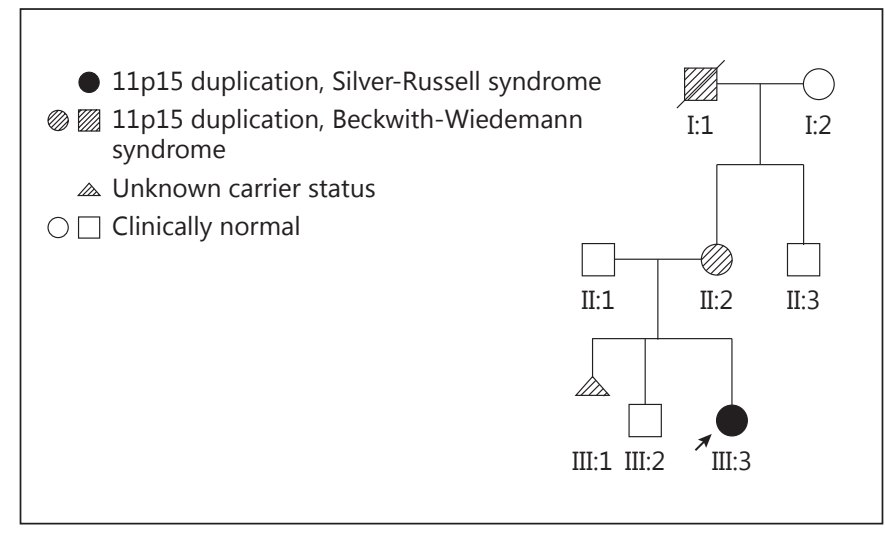

Fig. 1. Pedigree of the family with duplication of chromosome $11 \mathrm{p} 15$ that involves ICR1 and ICR2. Maternal inheritance of the duplication causes SRS, whereas paternal inheritance causes BWS.
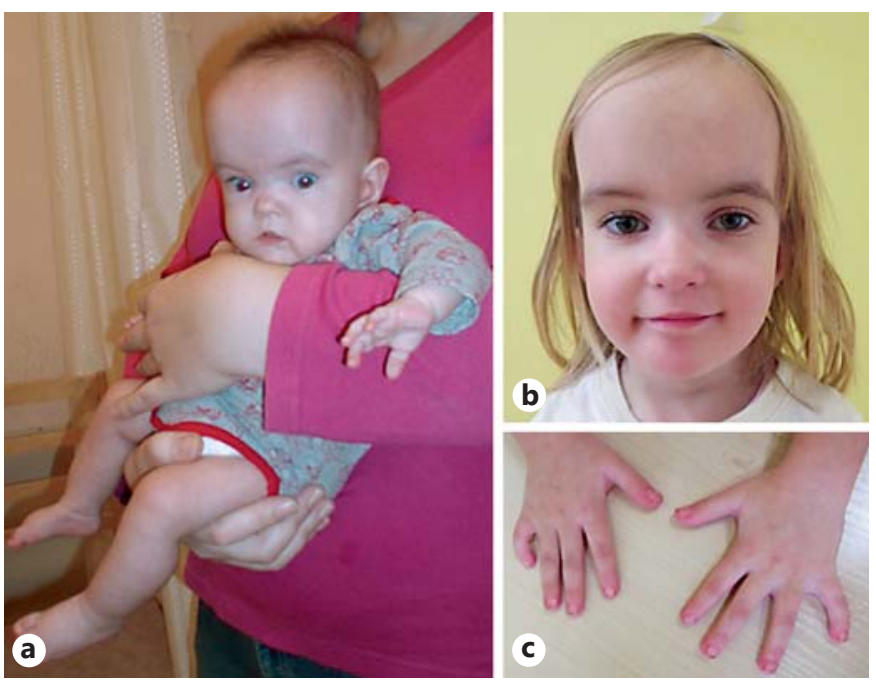

Fig. 2. The proband (III-3) at the age of 7 months (a) and at the age of 4.5 years (b). Note a relative macrocephaly, facial asymmetry, prominent forehead, and thin upper lip with downturned corners of the mouth. c Hands of the proband at the age of 4.5 years showing 5 th finger clinodactyly.

tions that affect both ICRs in $11 \mathrm{p} 15$ are known to be associated with growth retardation/SRS or overgrowth/ BWS depending on the parental origin of the imbalance [Begemann et al., 2012]. These duplications are mostly associated with translocations.

To our knowledge, we describe here the smallest familial pure $1.3-\mathrm{Mb}$ duplication in chromosomal region $11 \mathrm{p} 15.5 \mathrm{p} 15.4$ that involves both ICRs and is present in 3 generations causing an SRS or BWS phenotype.

\section{Case Report}

The proband (fig. 1, III:3), aged 5 years, was prematurely born at the 31 st gestational week via cesarean section due to intrauterine growth restriction, oligohydramnion, and fetal distress. Her prenatal course is also shortly described by Muru et al. [2013]. The fetal karyotype was normal $(46, \mathrm{XX})$, and no visible rearrangements were seen. Her birth weight was $890 \mathrm{~g}(-2.5 \mathrm{SD})$, birth length $34 \mathrm{~cm}(-3.5 \mathrm{SD})$, and head circumference $28 \mathrm{~cm}(-1 \mathrm{SD})$. SRS was clinically diagnosed as she also had body asymmetry, distinctive facial features (triangular face, prominent forehead, thin upper lip with downturned corners of the mouth) as well as dysmorphic ears, hypertelorism, blue sclerae, clinodactyly, and sandal groove of the toes (fig. 2a). Despite prematurity, her early postnatal period was normal. After birth, she had many respiratory infections, and in the second year of life, she was diagnosed with allergic asthma. As an infant, she had problems with gastroesophageal reflux and still has poor appetite. Her psychomotor development has been appropriate to age, although she has slight muscular hypotonia. She started walking independently when she was 17 months old, and she has good language skills. Because of astigmatism, she wears glasses.

At the age of 4.5 years, her height was $95.8 \mathrm{~cm}(-2.5 \mathrm{SD})$, weight $11.1 \mathrm{~kg}(-3.5 \mathrm{SD})$, and head circumference $51.1 \mathrm{~cm}(0 \mathrm{SD})$ (fig. $2 \mathrm{~b}$, c). The endocrinologist considered growth hormone therapy, but as the patient's growth velocity was appropriate $(4.8 \mathrm{~cm}$ within 6.5 months), a decision about the treatment was postponed.

The birth weight of the proband's mother (II:2) was 4,700 g $(+2.5 \mathrm{SD})$, and now her height is $182 \mathrm{~cm}(+3 \mathrm{SD})$. She has had the characteristic BWS facies since early childhood (fig. 3a, b) and also ear creases and pits. Her childhood passed without major problems. At the age of 10 years, she was diagnosed with latent diabetes, and now she has type 2 diabetes. She fulfilled 3 major diagnostic clinical criteria for BWS described by Weksberg et al. [2010]. She had 3 pregnancies, and the patient described here was her second delivery.

The brother of the patient (III:2) has a normal phenotype; his birth weight was $3,876 \mathrm{~g}$, and he has a normal postnatal growth.

The maternal grandfather's (I:1) height was $180 \mathrm{~cm}$ (0 SD), and he died of lung cancer at the age of 84 . We have the information about his phenotype only by photographs (fig. 3c). He had 2 major clinical criteria (macrosomia, positive family history) and 1 minor clinical criterion (characteristic facies) for BWS described by Weksberg et al. [2010].

\section{Material and Methods}

\section{Microarray Analysis}

DNA was extracted from peripheral blood of the proband and her mother and grandfather using a standard salting out procedure. Chromosomal microarray analysis (CMA) using Illumina HumanCytoSNP-12 BeadChips (Illumina Inc., San Diego, Calif., USA) was performed according to the manufacturer's protocol. Genotypes were called using GenomeStudio v2009.1 software (Illumina Inc.); $\log _{2} \mathrm{R}$ intensity ratio and $\mathrm{B}$-allele frequency values were extracted from GenomeStudio and used in subsequent DNA copy number analysis with quantiSNP v1.1 software using parameters suggested by the developers [Colella et al., 2007]. 
Fig. 3. The mother (II:2) in infancy (a) and at the age of 6 years (b). c The grandfather (I:1) at the age of 70 years. Note the characteristic BWS facies.
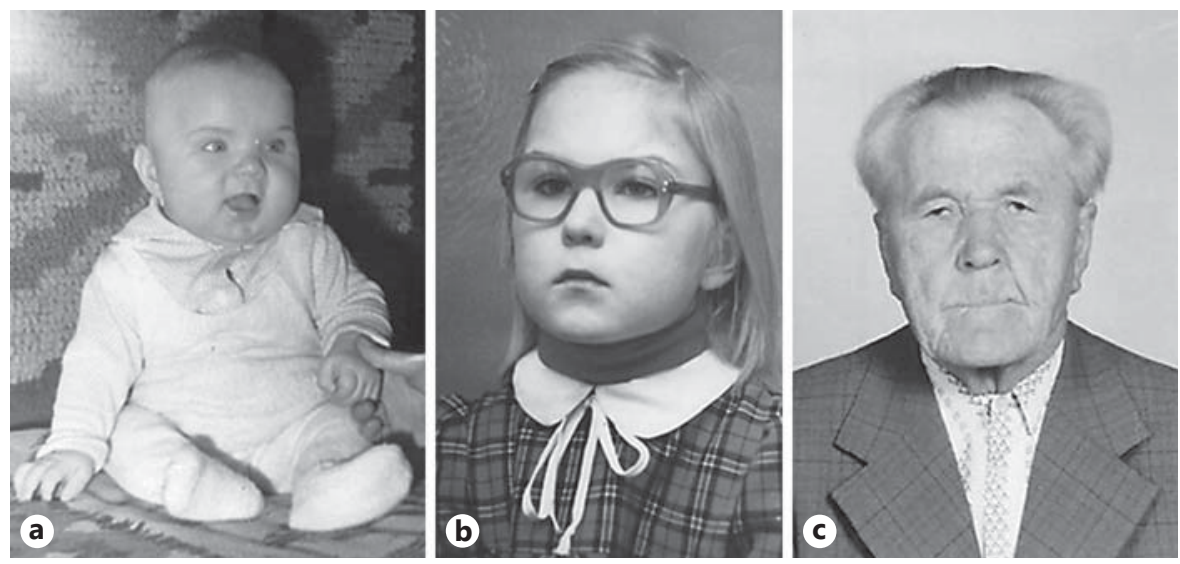

\section{Methylation Study}

Methylation-specific multiplex ligation-dependent probe amplification (MS-MLPA) was performed using SALSA ${ }^{\circledR}$ MS-MLPA ${ }^{\circledR}$ probe mixture ME030-B2 BWS/SRS (MRC-Holland, Amsterdam, The Netherlands) according to the manufacturer's instructions. PCR products were analyzed on a fluorescent capillary sequencer using the Genescan software (ABI 3130XL Genetic Analyzer, Applied Biosystems, Darmstadt, Germany). All MS-MLPA reactions were performed twice. MLPA data analysis was performed with the Coffalyser software (MRC-Holland).

\section{Results}

\section{Microarray Analysis}

In the proband, CMA revealed a $\sim 1.3-\mathrm{Mb}$ duplication on the short arm of chromosome 11 (arr[hg19] 11p15. $5 p 15.4(1,849,354-3,116,073) \times 3)$ that involves the ICR1 and ICR2 regions. The same duplication was also found in her mother and maternal grandfather and has been inherited within 3 generations in the same size and without rearrangements. We concluded that this is a tandem duplication.

\section{Methylation Study}

The MS-MLPA analysis of the proband, her mother, and maternal grandfather revealed an increase in copy number variation in altogether 26 MLPA probes in the whole analyzed $11 \mathrm{p} 15.5$ region. The mean ratio of these probes was $1.38(\mathrm{SD}=0.13)$. Probe ratios $>1.3$ are regarded as indicative of a heterozygous duplication (copy number change from 2 to 3 alleles). In the proband, a mild hypomethylation was detected in the ICR1 region, while in the ICR2 region hypermethylation was found. The proband's mother had ICR1 hypermethylation and ICR2 hypomethylation, and the maternal grandfather had also ICR1 hypermethylation and mild ICR2 hypomethylation.

\section{Discussion}

We report the familial case of an interstitial duplication of chromosome 11p15 which involves both ICR 1 and ICR2. To our knowledge, this is the first report of such a chromosomal rearrangement in $11 \mathrm{p} 15$ that is present in at least 3 generations. Although not described in many patients, maternal duplication of $11 \mathrm{p} 15$ is one possible cause of SRS with a frequency of $0.9 \%$ [Eggermann et al., 2014]. There are at least 13 studies reporting maternally inherited duplications as a cause of SRS. Most of such rearrangements involve ICR1 and ICR2 and are usually caused by unbalanced translocations, but duplication of the whole ICR2 as well as partial duplication of ICR1 can also cause SRS [Fisher et al., 2002; Eggermann et al., 2005; Schonherr et al., 2007; South et al., 2008; Bliek et al., 2009; Cardarelli et al., 2010; Eggermann et al., 2010; Bonaldi et al., 2011; Demars et al., 2011; Begemann et al., 2012; Chiesa et al., 2012; Hu et al., 2013; Brown et al., 2014].

In case of maternal inheritance, duplication of both ICR1 and ICR2 in $11 \mathrm{p} 15$ causes the SRS phenotype, whereas a paternally inherited similar duplication causes the BWS phenotype. Such familial parent-of-origin dependent opposite clinical syndromes have been reported also in other studies, but compared to our report, their genetic causes have been different (unbalanced translocations causing duplication of $11 \mathrm{p} 15$ and between-arm intrachromosomal insertion of 11p15.5) [South et al., 2008; Bliek et al., 2009; Cardarelli et al., 2010; Brown et al., 2014]. In our proband, interstitial duplication of 11 p15 shows hypomethylation in ICR1 and hypermethylation in ICR2. Therefore, there are 2 active copies of CDKN1C and H19, which is compatible with either maternal uniparental disomy or maternal duplication in $11 \mathrm{p} 15$ [Chiesa et al., 2012]. Hence, the SRS phenotype in the proband 
reflects an increased expression of the maternally expressed CDKN1C. The BWS phenotype in the mother and maternal grandfather is caused by overexpression of IGF2. Increased dosage of paternally expressed genes is caused by ICR1 hypermethylation.

It is very difficult to compare our patient with previously reported SRS patients with maternally inherited duplications. First, the data describe variable phenotypes even if the molecular cause was similar. Secondly, the phenotype of patients with duplications that are caused by unbalanced translocations might be influenced by accompanying chromosomal imbalances [South et al., 2008; Bliek et al., 2009; Hu et al., 2013]. Chiesa et al. [2012] described a patient with a de novo maternal 1.2-Mb inverted duplication and classical SRS phenotype, who molecularly and clinically most resembles our proband. Unfortunately, we have not done FISH analysis in our proband.

For the clinical diagnosis of SRS in our proband, we used the Bartholdi scoring system [Bartholdi et al., 2009]. She met 11 positive criteria out of 15 , including pre- and postnatal growth failure, body asymmetry, classical facial phenotype, and F5 clinodactyly. This relatively high score and classical findings of SRS might be explained by the fact that the interstitial duplication involves only imprinted regions. It also explains her age-appropriate psychomotor development, which is usually normal among SRS patients with primary imprinting disorders [Kotzot, 2008; Wakeling et al., 2010; Demars et al., 2011].
Contrary to the patient's classical SRS phenotype, the BWS clinical picture of her mother and grandfather has been probably quite mild and has become less evident in adulthood as described among adults with BWS [Weksberg et al., 2010]. Their childhood passed without major problems (e.g. childhood tumors). Unfortunately, we cannot determine the origin of the duplication further in this family, but the proband's maternal grandfather must have carried his duplication on a paternally inherited chromosome 11, and this might be either inherited or de novo in origin.

In conclusion, although maternal duplications involving $11 \mathrm{p} 15$ are rare, they must be considered when copy number variation together with an aberrant methylation pattern in ICR1 and ICR2 at $11 \mathrm{p} 15$ is found. This should lead to further genetic testing to detect the cause of such chromosomal rearrangement, and it might reveal new information about previous generations who have not been suspected to have a clinical syndrome.

\section{Acknowledgements}

We thank the patient and her family. This work was supported by grant GARLA8175 and GARLA0355P from the Estonian Science Foundation. Two authors (T.K. and K.Õ.) are members of the European Network of Congenital Imprinting Disorders (EUCID. net), which is supported by COST (BM1208).

\section{References}

- Bartholdi D, Krajewska-Walasek M, Ounap K, Gaspar H, Chrzanowska KH, et al: Epigenetic mutations of the imprinted IGF2-H19 domain in Silver-Russell syndrome (SRS): results from a large cohort of patients with SRS and SRS-like phenotypes. J Med Genet 46: 192-197 (2009).

-Begemann M, Spengler S, Gogiel M, Grasshoff U, Bonin $\mathrm{M}$, et al: Clinical significance of copy number variations in the $11 \mathrm{p} 15.5$ imprinting control regions: new cases and review of the literature. J Med Genet 49:547-553 (2012).

Bliek J, Snijder S, Maas SM, Polstra A, van der Lip $\mathrm{K}$, et al: Phenotypic discordance upon paternal or maternal transmission of duplications of the 11p15 imprinted regions. Eur J Med Genet 52:404-408 (2009).

Bonaldi A, Mazzeu JF, Costa SS, Honjo RS, Bertola DR, et al: Microduplication of the ICR2 domain at chromosome $11 \mathrm{p} 15$ and familial Silver-Russell syndrome. Am J Med Genet A 155A:2479-2483 (2011).
Brown LA, Rupps R, Penaherrera MS, Robinson WP, Patel MS, et al: A cryptic familial rearrangement of $11 \mathrm{p} 15.5$, involving both imprinting centers, in a family with a history of short stature. Am J Med Genet A 164A:15871594 (2014).

Cardarelli L, Sparago A, De Crescenzo A, Nalesso E, Zavan B, et al:Silver-Russell syndrome and Beckwith-Wiedemann syndrome phenotypes associated with $11 \mathrm{p}$ duplication in a single family. Pediatr Dev Pathol 13:326-330 (2010).

-Chiesa N, De Crescenzo A, Mishra K, Perone L, Carella M, et al: The KCNQ1OT1 imprinting control region and non-coding RNA: new properties derived from the study of Beckwith-Wiedemann syndrome and Silver-Russell syndrome cases. Hum Mol Genet 21:1025 (2012).

Colella S, Yau C, Taylor JM, Mirza G, Butler H, et al: QuantiSNP: an Objective Bayes HiddenMarkov Model to detect and accurately map copy number variation using SNP genotyping data. Nucleic Acids Res 35:2013-2025 (2007).
Demars J, Rossignol S, Netchine I, Lee KS, Shmela M, et al: New insights into the pathogenesis of Beckwith-Wiedemann and Silver-Russell syndromes: contribution of small copy number variations to $11 \mathrm{p} 15$ imprinting defects. Hum Mutat 32:1171-1182 (2011).

Eggermann T, Meyer E, Obermann C, Heil I, Schuler $\mathrm{H}$, et al: Is maternal duplication of 11 p15 associated with Silver-Russell syndrome? J Med Genet 42:e26 (2005).

- Eggermann T, Eggermann K, Schonherr N: Growth retardation versus overgrowth: Silver-Russell syndrome is genetically opposite to Beckwith-Wiedemann syndrome. Trends Genet 24:195-204 (2008).

Eggermann T, Spengler S, Bachmann N, Baudis M, Mau-Holzmann UA, et al: Chromosome 11 p15 duplication in Silver-Russell syndrome due to a maternally inherited translocation $\mathrm{t}(11 ; 15)$. Am J Med Genet A 152A:1484-1487 (2010). 
Eggermann T, Heilsberg AK, Bens S, Siebert R, Beygo J, et al: Additional molecular findings in 11p15-associated imprinting disorders: an urgent need for multi-locus testing. J Mol Med (Berl) 92:769-777 (2014).

Fisher AM, Thomas NS, Cockwell A, Stecko O, Kerr B, et al: Duplications of chromosome 11 p15 of maternal origin result in a phenotype that includes growth retardation. Hum Genet 111:290-296 (2002).

Fokstuen S, Kotzot D: Chromosomal rearrangements in patients with clinical features of Silver-Russell syndrome. Am J Med Genet A 164A:1595-1605 (2014).

-Gicquel C, Rossignol S, Cabrol S, Houang M, Steunou V, et al: Epimutation of the telomeric imprinting center region on chromosome 11 p15 in Silver-Russell syndrome. Nat Genet 37:1003-1007 (2005).
Hu J, Sathanoori M, Kochmar S, Madan-Khetarpal S, McGuire M, Surti U: Co-existence of 9p deletion and Silver-Russell syndromes in a patient with maternally inherited cryptic complex chromosome rearrangement involving chromosomes 4, 9, and 11. Am J Med Genet A 161A:179-184 (2013).

Kotzot D: Maternal uniparental disomy 7 and Silver-Russell syndrome - clinical update and comparison with other subgroups. Eur J Med Genet 51:444-451 (2008).

Muru K, Vals MA, Sitska M, Asser K, Tammur P, et al: Outcome of children with marked changes in maternal screening tests and normal karyotype. Genetics 3:123 (2013).

Schonherr N, Meyer E, Roos A, Schmidt A, Wollmann HA, Eggermann T: The centromeric $11 \mathrm{p} 15$ imprinting centre is also involved in Silver-Russell syndrome. J Med Genet 44:5963 (2007).
South ST, Whitby H, Maxwell T, Aston E, Brothman AR, Carey JC: Co-occurrence of $4 \mathrm{p} 16.3$ deletions with both paternal and maternal duplications of 11p15: modification of the WolfHirschhorn syndrome phenotype by genetic alterations predicted to result in either a Beckwith-Wiedemann or Russell-Silver phenotype. Am J Med Genet A 146A:2691-2697 (2008).

Wakeling EL, Amero SA, Alders M, Bliek J, Forsythe E, et al: Epigenotype-phenotype correlations in Silver-Russell syndrome. J Med Genet 47:760-768 (2010).

Weksberg R, Shuman C, Beckwith JB: BeckwithWiedemann syndrome. Eur J Hum Genet 18: 8-14 (2010). 